\title{
Correction: Early symptoms in symptomatic and preclinical genetic frontotemporal lobar degeneration
}

Tavares TP, Mitchell DGV, Coleman KK, et al. Early symptoms in symptomatic and preclinical genetic frontotemporal lobar degeneration. J Neurol Neurosurg \& Psychiatry 2020;91:97584. doi: 10.1136/jnnp-2020-322987.

The Genetic FTD Initiative, (GENFI) should have been listed in the paper.

List of GENFI Consortium Members

- Alazne Gabilondo - Neuroscience Area, Biodonostia Health Research Insitute, San Sebastian, Gipuzkoa, Spain

- Albert Lladó - Alzheimer's disease and Other Cognitive Disorders Unit, Neurology Service, Hospital Clínic, Barcelona, Spain

- Alessandro Padovani - Centre for Neurodegenerative Disorders, Neurology Unit, Department of Clinical and Experimental Sciences, University of Brescia, Brescia, Italy

- Ana Gorostidi - Neuroscience Area, Biodonostia Health Research Insitute, San Sebastian, Gipuzkoa, Spain

- Ana Verdelho - Department of Neurosciences and Mental Health, Centro Hospitalar Lisboa Norte - Hospital de Santa Maria \& Faculty of Medicine, University of Lisbon, Lisbon, Portugal

- Andrea Arighi - Fondazione IRCCS Ca' Granda Ospedale Maggiore Policlinico, Neurodegenerative Diseases Unit, Milan, Italy; University of Milan, Centro Dino Ferrari, Milan, Italy

- Anna Antonell - Alzheimer's disease and Other Cognitive Disorders Unit, Neurology Service, Hospital Clínic, Barcelona, Spain

- Beatriz Santiago - Neurology Department, Centro Hospitalar e Universitario de Coimbra, Coimbra, Portugal

- Begoña Indakoetxea - Cognitive Disorders Unit, Department of Neurology, Donostia University Hospital, San Sebastian, Gipuzkoa, Spain; Neuroscience Area, Biodonostia Health Research Insitute, San Sebastian, Gipuzkoa, Spain

- Benedetta Nacmias - Department of Neuroscience, Psychology, Drug Research and Child Health, University of Florence, Florence, Italy

- Benjamin Bender - Department of Diagnostic and Interventional Neuroradiology, University of Tübingen, Tübingen, Germany

- Camilla Ferrari - Department of Neuroscience, Psychology, Drug Research and Child Health, University of Florence, Florence, Italy

- Carlo Wilke - Department of Neurodegenerative Diseases, Hertie-Institute for Clinical Brain Research and Center of Neurology, University of Tübingen, Tübingen, Germany; Center for Neurodegenerative Diseases (DZNE), Tübingen, Germany

- Carolin Heller - Dementia Research Centre, Department of Neurodegenerative Disease, UCL Institute of Neurology, Queen Square, London, UK

- Carolina Maruta - Laboratory of Language Research, Centro de Estudos Egas Moniz, Faculty of Medicine, University of Lisbon, Lisbon, Portugal

- Caroline Greaves - Dementia Research Centre, Department of Neurodegenerative Disease, UCL Institute of Neurology, Queen Square, London, UK

- Carolyn Timberlake - Department of Clinical Neurosciences, University of Cambridge, Cambridge, UK

- Catarina B. Ferreira - Laboratory of Neurosciences, Institute of Molecular Medicine, Faculty of Medicine, University of Lisbon, Lisbon, Portugal

- Catharina Prix - Neurologische Klinik, Ludwig-Maximilians-Universität München, Munich, Germany

- Chiara Fenoglio - Fondazione IRCCS Ca' Granda Ospedale Maggiore Policlinico, Neurodegenerative Diseases Unit, Milan, Italy; University of Milan, Centro Dino Ferrari, Milan, Italy

- Christin Andersson - Department of Clinical Neuroscience, Karolinska Institutet, Stockholm, Sweden

- Cristina Polito - Department of Biomedical, Experimental and Clinical Sciences "Mario Serio", Nuclear Medicine Unit, University of Florence, Florence, Italy

- David Cash - Dementia Research Centre, Department of Neurodegenerative Disease, UCL Institute of Neurology, Queen Square, London, UK 
- David L Thomas - Neuroimaging Analysis Centre, Department of Brain Repair and Rehabilitation, UCL Institute of Neurology, Queen Square, London, UK

- David Tang-Wai - The University Health Network, Krembil Research Institute, Toronto, Canada

- Diana Duro - Faculty of Medicine, University of Coimbra, Coimbra, Portugal

- Ekaterina Rogaeva - Tanz Centre for Research in Neurodegenerative Diseases, University of Toronto, Toronto, Canada

- Elio Scarpini - Fondazione IRCCS Ca' Granda Ospedale Maggiore Policlinico, Neurodegenerative Diseases Unit, Milan, Italy; University of Milan, Centro Dino Ferrari, Milan, Italy

- Elisa Semler - Department of Neurology, University of Ulm, Ulm

- Elisabeth Wlasich - Neurologische Klinik, Ludwig-Maximilians-Universität München, Munich, Germany

- Emily Todd - Department of Neurodegenerative Disease, UCL Institute of Neurology, UK

- Enrico Premi - Centre for Neurodegenerative Disorders, Neurology Unit, Department of Clinical and Experimental Sciences, University of Brescia, Brescia, Italy

- Gabriel Miltenberger - Faculty of Medicine, University of Lisbon, Lisbon, Portugal

- Gemma Lombardi - Department of Neuroscience, Psychology, Drug Research and Child Health, University of Florence, Florence, Italy

- Georgia Peakman - Department of Neurodegenerative Disease, UCL Institute of Neurology, UK

- Giacomina Rossi - Fondazione IRCCS Istituto Neurologico Carlo Besta, Milano, Italy

- Giorgio Fumagalli - Fondazione IRCCS Ca' Granda Ospedale Maggiore Policlinico, Neurodegenerative Diseases Unit, Milan, Italy; University of Milan, Centro Dino Ferrari, Milan, Italy; Department of Neurosciences, Psychology, Drug Research and Child Health (NEUROFARBA), University of Florence, Florence, Italy

- Giorgio Giaccone - Fondazione IRCCS Istituto Neurologico Carlo Besta, Milano, Italy

- Giuliano Binetti - Istituto di Ricovero e Cura a Carattere Scientifico Istituto Centro San Giovanni di Dio Fatebenefratelli, Brescia, Italy

- Giuseppe Di Fede - Fondazione IRCCS Istituto Neurologico Carlo Besta, Milano, Italy

- Hakan Thonberg - Centre for Alzheimer Research, Division of Neurogeriatrics, Karolinska Institutet, Stockholm, Sweden

- Hans-Otto Karnath - Division of Neuropsychology, Hertie-Institute for Clinical Brain Research and Centre of Neurology, University of Tübingen, Tübingen, Germany

- Henrik Zetterberg - Dementia Research Institute, Department of Neurodegenerative Disease, UCL Institute of Neurology, Queen Square, London, UK

- Ione Woollacott - Dementia Research Centre, Department of Neurodegenerative Disease, UCL Institute of Neurology, Queen Square, London, UK

- Janne Papma - Department of Neurology, Erasmus Medical Centre, Rotterdam

- Jason Warren - Dementia Research Centre, Department of Neurodegenerative Disease, UCL Institute of Neurology, Queen Square, London, UK

- Jaume Olives - Alzheimer's disease and Other Cognitive Disorders Unit, Neurology Service, Hospital Clínic, Barcelona, Spain

- Jennifer Nicholas - Department of Medical Statistics, London School of Hygiene and Tropical Medicine, London, UK

- Jessica Panman - Department of Neurology, Erasmus Medical Centre, Rotterdam, Netherlands

- Jorge Villanua - OSATEK, University of Donostia, San Sebastian, Gipuzkoa, Spain

- Jose Bras - Dementia Research Institute, Department of Neurodegenerative Disease, UCL Institute of Neurology, Queen Square, London, UK

- Katrina Moore - Dementia Research Centre, Department of Neurodegenerative Disease, UCL Institute of Neurology, Queen Square, London UK

- Lieke Meeter - Department of Neurology, Erasmus Medical Centre, Rotterdam, Netherlands

- Linn Öijerstedt - Department of Geriatric Medicine, Karolinska University HospitalHuddinge, Stockholm, Sweden

- Lize Jiskoot - Department of Neurology, Erasmus Medical Centre, Rotterdam, Netherlands

- Luisa Benussi - Istituto di Ricovero e Cura a Carattere Scientifico Istituto Centro San Giovanni di Dio Fatebenefratelli, Brescia, Italy

- María de Arriba - Neuroscience Area, Biodonostia Health Research Insitute, San Sebastian, Gipuzkoa, Spain

- Maria João Leitão - Centre of Neurosciences and Cell Biology, Universidade de Coimbra, Coimbra, Portugal

- Maria Rosario Almeida - Faculty of Medicine, University of Coimbra, Coimbra, Portugal 
- Martin Rosser - Dementia Research Centre, Department of Neurodegenerative Disease, UCL Institute of Neurology, Queen Square, London, UK

- Martina Bocchetta - Dementia Research Centre, Department of Neurodegenerative Disease, UCL Institute of Neurology, Queen Square, London, UK

- Mathieu Vandenbulcke - Geriatric Psychiatry Service, University Hospitals Leuven, Belgium; Neuropsychiatry, Department of Neurosciences, KU Leuven, Leuven, Belgium

- Maura Cosseddu - Centre for Neurodegenerative Disorders, Neurology Unit, Department of Clinical and Experimental Sciences, University of Brescia, Brescia, Italy

- Michela Pievani - Istituto di Ricovero e Cura a Carattere Scientifico Istituto Centro San Giovanni di Dio Fatebenefratelli, Brescia, Italy

- Michele Veldsman - Nuffield Department of Clinical Neurosciences, Medical Sciences Division, University of Oxford, Oxford, UK

- Miguel Castelo-Branco - Faculty of Medicine, University of Coimbra, Coimbra, Portugal

- Miguel Tábuas-Pereira - Neurology Department, Centro Hospitalar e Universitario de Coimbra, Coimbra, Portugal

- Mikel Tainta - Neuroscience Area, Biodonostia Health Research Insitute, San Sebastian, Gipuzkoa, Spain

- Mircea Balasa - Alzheimer's disease and Other Cognitive Disorders Unit, Neurology Service, Hospital Clínic, Barcelona, Spain

- Miren Zulaica - Neuroscience Area, Biodonostia Health Research Insitute, San Sebastian, Gipuzkoa, Spain

- Morris Freedman - Baycrest Health Sciences, Rotman Research Institute, University of Toronto, Toronto, Canada

- Myriam Barandiaran - Cognitive Disorders Unit, Department of Neurology, Donostia University Hospital, San Sebastian, Gipuzkoa, Spain; Neuroscience Area, Biodonostia Health Research Insitute, San Sebastian, Gipuzkoa, Spain

- Nick Fox - Dementia Research Centre, Department of Neurodegenerative Disease, UCL Institute of Neurology, Queen Square, London, UK

- Nuria Bargalló - Imaging Diagnostic Centre, Hospital Clínic, Barcelona, Spain

- Paola Caroppo - Fondazione IRCCS Istituto Neurologico Carlo Besta, Milano, Italy

- Pedro Rosa-Neto - Translational Neuroimaging Laboratory, McGill Centre for Studies in Ageing, McGill University, Montreal, Québec, Canada

- Philip Vandamme - Neurology Service, University Hospitals Leuven, Belgium; Laboratory for Neurobiology, VIB-KU Leuven Centre for Brain Research, Leuven, Belgium

- Pietro Tiraboschi - Fondazione IRCCS Istituto Neurologico Carlo Besta, Milano, Italy

- Rachelle Shafei - Dementia Research Centre, Department of Neurodegenerative Disease, UCL Institute of Neurology, Queen Square, London, UK

- Rhian Convery - Dementia Research Centre, Department of Neurodegenerative Disease, UCL Institute of Neurology, Queen Square, London, UK

- Ricardo Taipa - Neuropathology Unit and Department of Neurology, Centro Hospitalar do Porto - Hospital de Santo António, Oporto, Portugal

- Rick van Minkelen - Department of Clinical Genetics, Erasmus Medical Centre, Rotterdam, Netherlands

- Rita Guerreiro - Dementia Research Institute, Department of Neurodegenerative Disease, UCL Institute of Neurology, Queen Square, London, UK

- Robart Bartha - Department of Medical Biophysics, The University of Western Ontario, London, Ontario, Canada; Centre for Functional and Metabolic Mapping, Robarts Research Institute, The University of Western Ontario, London, Ontario, Canada

- Roberto Gasparotti - Neuroradiology Unit, University of Brescia, Brescia, Italy

- Ron Keren - The University Health Network, Toronto Rehabilitation Institute, Toronto, Canada

- Rosa Rademakers - Department of Neurosciences, Mayo Clinic, Jacksonville, Florida, USA

- Rose Bruffaerts - Laboratory for Cognitive Neurology, Department of Neurosciences, KU Leuven, Leuven, Belgium

- Sandra Black - Sunnybrook Health Sciences Centre, Sunnybrook Research Institute, University of Toronto, Toronto, Canada

- Sandra Loosli - Neurologische Klinik, Ludwig-Maximilians-Universität München, Munich, Germany

- Sara Mitchell - Sunnybrook Health Sciences Centre, Sunnybrook Research Institute, University of Toronto, Toronto, Canada

- Sara Prioni - Fondazione IRCCS Istituto Neurologico Carlo Besta, Milano, Italy

- Sarah Anderl-Straub - Department of Neurology, University of Ulm, Ulm, Germany

- Sebastien Ourselin - School of Biomedical Engineering \& Imaging Sciences, King's College London, London, UK 
- Serge Gauthier - Alzheimer Disease Research Unit, McGill Centre for Studies in Ageing, Department of Neurology \& Neurosurgery, McGill University, Montreal, Québec, Canada

- Sergi Borrego-Ecija - Alzheimer's disease and Other Cognitive Disorders Unit, Neurology Service, Hospital Clínic, Barcelona, Spain

- Silvana Archetti - Biotechnology Laboratory, Department of Diagnostics, Spedali Civili Hospital, Brescia, Italy

- Simon Mead - MRC Prion Unit, Department of Neurodegenerative Disease, UCL Institute of Neurology, Queen Square, London, UK

- Sónia Afonso - Instituto Ciencias Nucleares Aplicadas a Saude, Universidade de Coimbra, Coimbra, Portugal

- Sonja Schönecker - Neurologische Klinik, Ludwig-Maximilians-Universität München, Munich, Germany

- Stefano Gazzina - Centre for Neurodegenerative Disorders, Neurology Unit, Department of Clinical and Experimental Sciences, University of Brescia, Brescia, Italy

- Thomas Cope - Department of Clinical Neuriscience, University of Cambridge, Cambridge, UK

- Tim Rittman - Department of Clinical Neurosciences, University of Cambridge, Cambridge, UK

- Tobias Hoegen - Neurologische Klinik, Ludwig-Maximilians-Universität München, Munich, Germany

- Toby Flanagan - Faculty of Biology, Medicine and Health, Division of Neuroscience and Experimental Psychology, University of Manchester, Manchester, UK

- Valentina Bessi - Department of Neuroscience, Psychology, Drug Research and Child Health, University of Florence, Florence, Italy

- Veronica Redaelli - Fondazione IRCCS Istituto Neurologico Carlo Besta, Milano, Italy

- Vesna Jelic - Division of Clinical Geriatrics, Karolinska Institutet, Stockholm, Sweden

- Yolande Pijnenburg - Amsterdam University Medical Centre, Amsterdam VUmc, Amsterdam, Netherlands

- Zigor Díaz - CITA Alzheimer, San Sebastian, Gipuzkoa, Spain

(C) Author(s) (or their employer(s)) 2020. No commercial re-use. See rights and permissions. Published by BMJ.

J Neurol Neurosurg Psychiatry 2020;91:e3. doi:10.1136/jnnp-2020-322987corr1

D Check for updates 